Check for updates

Cite this: RSC Adv., 2020, 10, 28032

Received 22nd June 2020

Accepted 15th July 2020

DOI: 10.1039/d0ra05471b

rsc.li/rsc-advances

\section{Thermo-responsive wettability via surface roughness change on polymer-coated titanate nanorod brushes toward fast and multi-directional droplet transport $\uparrow$}

\author{
Kenji Okada, (D) *ab Yoko Miura, ${ }^{a}$ Tomoya Chiya, ${ }^{a}$ Yasuaki Tokudome (D) a \\ and Masahide Takahashi iD *a
}

\begin{abstract}
A novel approach for thermo-responsive wettability has been accomplished by surface roughness change induced by thermal expansion of paraffin coated on titanate nanostructures. The surface exhibits thermoresponsive and reversible wettability change in a hydrophobic regime; the surface shows superhydrophobicity with contact angles of $\sim 157^{\circ}$ below $50{ }^{\circ} \mathrm{C}$ and $\sim 118^{\circ}$ above $50{ }^{\circ} \mathrm{C}$ due to a decrease of surface roughness caused by thermally-expanded paraffin at higher temperatures. Reversible wettability change of $\sim 40^{\circ}$ of a contact angle allows for fast and multi-directional droplet transport. The present approach affords a versatile selection of materials and wide variety of contact angles, promoting both scientific advancement and technology innovation in the field of smart surfaces.
\end{abstract}

A smart surface with stimuli-responsive wettability is of great importance for various applications including self-cleaning, oil/ water separation, water motion, droplet microfluidics, biochemical analysis and drug delivery. ${ }^{1-5}$ In general, such smart surfaces can be achieved by the use of responsive molecules or polymers that can reversibly switch their chemical transformation and polarity ${ }^{6}$ by external stimuli such as light, ${ }^{7}$ $\mathrm{pH},{ }^{8,9}$ electric field, ${ }^{10}$ magnetic field, ${ }^{11}$ and temperature. ${ }^{\mathbf{1 2 , 1 3}}$ Among them, a thermo-responsive smart surface has aroused paramount interest as temperature is the most common and facile stimulus for practical applications. ${ }^{14-17}$ Poly $(N$-isopropylacrylamide) (PNIPAAm), that shows a lower critical solution temperature (LCST) phase transition at about $32-33^{\circ} \mathrm{C}$, has been typically used to fabricate thermo-responsive smart surfaces. The polymer chains are hydrated to become swelling states in water below LCST, and dehydrated to become compact structures when it is heated up above LCST. An increase of temperature causes a change of chemical interaction (hydrogen bonding interaction) at a surface/water interface to being weaken, resulting in the change of surface wettability from a hydrophilic state to a hydrophobic state. As similarly to the PNIPAAm based thermo-responsive smart surface, most of thermo-responsive smart surfaces exhibit a higher

${ }^{a}$ Department of Materials Science, Graduate School of Engineering, Osaka Prefecture University, Sakai, Osaka, 599-8531, Japan.E-mail: okada@photomater.com; masa@ photomater.com

${ }^{b} J S T$, PRESTO, 4-1-8 Honcho, Kawaguchi, Saitama, 332-0012, Japan

$\dagger$ Electronic supplementary information (ESI) available. See DOI: 10.1039/d0ra05471b hydrophobicity at higher temperatures because chemical interaction (e.g., hydrogen bonding interaction) between polymer and water becomes weaker. Although reversibly regulated surface wettability from a hydrophobic state to a hydrophilic state with increasing temperature has been recently reported by using upper critical solution temperature-type (UCST-type) polymers, there is still few reports on the fabrication of such surface. It is rather difficult to achieve polymers being hydrophilic at higher temperatures. ${ }^{\mathbf{1 4}}$ Apart from thermo-responsive smart surface, there is another important issue to be discussed from a scientific perspective for the stimuli-responsive wettability. Most of stimuli-responsive wettability demonstrated so far exclusively rely on the use of chemical transformation of responsive polymers. ${ }^{18}$ From a technological perspective, chemical interaction change at a surface/water interface might affect components in liquid, that would cause problems in some applications such as micro-fluidic control and drug delivery. To explore the fabrication protocol of stimuliresponsive wettability based on a novel approach will promote scientific advancement and technology innovation in the field of smart surfaces.

In the present study, we report a novel concept for thermoresponsive wettability, that exploits surface roughness change induced by thermal expansion of polymers on nanostructured inorganic substrates (Fig. 1). According to Wenzel and CassieBaxter models, the surface wettability for water is determined by not only chemical interaction at a surface/water interface but also surface roughness. ${ }^{19-22}$ When the chemical interaction is constant, a decrease of surface roughness leads to reduced hydrophobicity (in the case of Young contact angle, the water 


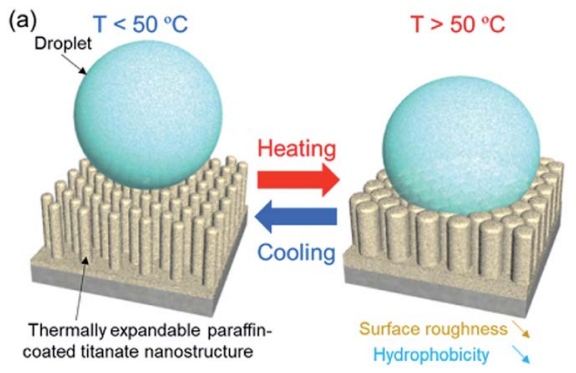

(b)

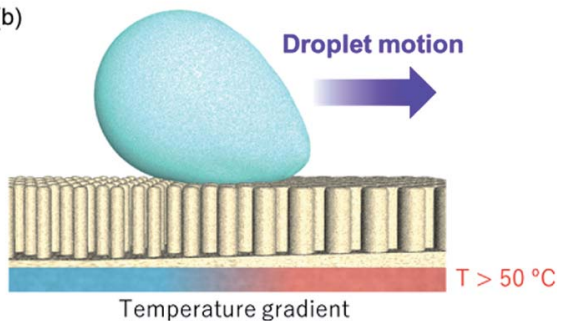

Fig. 1 (a) A concept of the present study for thermo-responsive wettability induced by surface roughness change. (b) A droplet transport by spatially heating the substrate at around room temperature where the temperature gradient of several degrees induces a droplet motion from a cooler part to a hotter part.

contact angle (CA) at the perfectly flat and rigid surface, higher than $90^{\circ}$ ), while opposite trend is observed for the hydrophilic surface. This indicates that the stimuli-responsive surface wettability could be realized by changing only surface roughness without any change on the chemical interaction. To this end, we have prepared paraffin-coated inorganic nanostructures where paraffin, that shows around 10\% thermal expansion with melting, contributes to surface roughness change by heating and the inorganic (titanate) nanostructures provide a homogeneously rough surface. Paraffin has been widely employed in a linear actuator device "wax motors" as it shows a drastic change on volume by heating/cooling. ${ }^{23-25}$ In a case of the present paraffin-coated inorganic nanostructures, the surface shows high hydrophobicity at low temperature (room temperature). While, at high temperature paraffin partially fills up the gap between the nanostructures by a thermal expansion, resulting in reduced hydrophobicity due to a decrease of surface roughness (Fig. 1a). In the present system, the chemical interaction at a surface/water interface remains unchanged upon heating because paraffin, that is an alkane with a chemical formula $\mathrm{C}_{n} \mathrm{H}_{2 n+2}$, exhibits no chemical transformation. The surface wettability can be reversibly controlled only by a change of surface roughness. In addition, the paraffin-coated inorganic nanostructures can afford a droplet transport by partially heating the substrate at room temperature where the temperature gradient induces a droplet motion from an unheated point to a heated point by a wettability gradient (Fig. 1b). Partial heating at room temperature is relatively simple and uncomplicated and requires less energy compared to partial cooling of a heated substrate. Furthermore, this novel system, that is unaccompanied by a change of chemical interaction at a surface/water interface, allows for a droplet transport without any change of the chemical component of the droplet; in droplet transport systems that rely on chemical transformation of responsive materials, the change on surface chemical properties might affect components in a droplet (e.g., trapping ionic species on the surface).

Vertically-aligned titanate nanorods on a titanium plate (TNR brush) were employed as inorganic nanostructures, that was prepared according to the previously reported procedure. ${ }^{26-28}$ The surface of TNR brush was modified with octyltrimethoxysilane (OTMS) by chemical vapor deposition in order to improve a chemical interaction with paraffin. Then, paraffin (melting point at $42-44{ }^{\circ} \mathrm{C}$ ) dissolved in isooctane was spincoated on the OTMS-modified TNR brush to obtain a paraffincoated TNR brush (hereafter the sample is denoted as paraffin/TNR brush). The pristine TNR brush exhibited superhydrophilicity with a CA of $\sim 0^{\circ}$ due to the high surface roughness by the nanostructures of hydrophilic titanate. In contrast, the OTMS-modified TNR brush and paraffin/TNR brush showed superhydrophobicity with CAs above $150^{\circ}$, indicating the successful OTMS modification and paraffin coating with maintaining the surface nanostructures. The OTMS modification and the paraffin-coating were also confirmed by Fouriertransform infrared spectroscopy (FT-IR) (see Fig. S1 †). Fig. 2a exhibits a scanning electron microscope (SEM) image of the paraffin/TNR brush. A comparison with SEM images of the pristine TNR brush confirms a homogeneous coating of paraffin with $\sim 23 \mathrm{~nm}$ in thickness on the surface of TNR brush (Fig. 2b and see Fig. S2 $\dagger$ ). The wettability of the paraffin/TNR brush was investigated at different substrate temperatures (Fig. 2c). The temperature of substrate was monitored by an infrared thermography camera. As shown in the Fig. 2c, the paraffin/TNR brush exhibited superhydrophobicity with a CA of $\sim 157^{\circ}$ below $50{ }^{\circ} \mathrm{C}$ and the surface drastically changed to a less hydrophobic state with a $\mathrm{CA}$ of $\sim 118^{\circ}$ above $50^{\circ} \mathrm{C}$. The change of wettability was reversible at lower/higher temperature than $50{ }^{\circ} \mathrm{C}$ (Fig. 2d). The temperature failure of nanoscale and
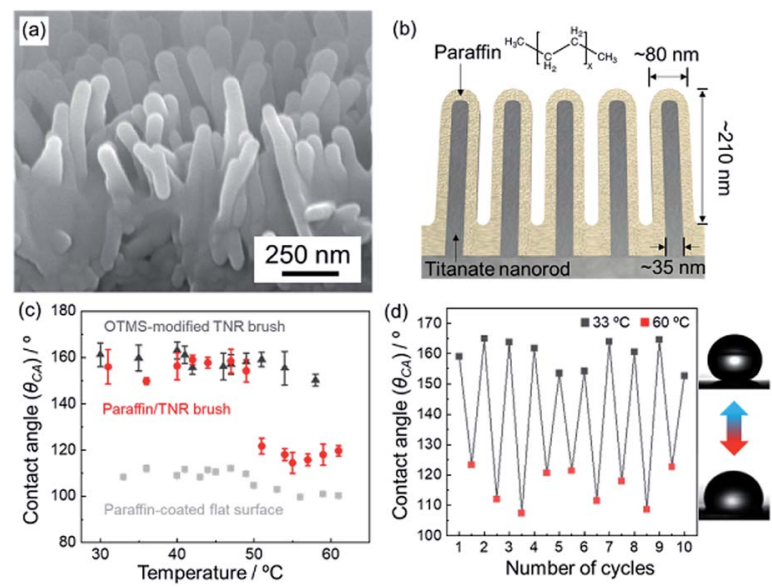

Fig. 2 Cross-sectional SEM image (a) and the corresponding illustration (b) of the paraffin/TNR brush. (c) Temperature-dependence of the CAs on the paraffin/TNR brush, OTMS-modified TNR brush and the paraffin-coated flat surface. (d) Reversible change of wettability (CA) on the paraffin/TNR brush at $33^{\circ} \mathrm{C}$ (black) and $60{ }^{\circ} \mathrm{C}$ (red). 
macroscale structures was not observed in this temperature range (Fig. S3 and $\mathrm{S} 4 \dagger$ ). It should be mentioned that the wettability of paraffin-coated flat surface (paraffin-coated glass) nearly unchanged at different temperatures (the small change of wettability is presumably due to the formation of a lubricantinfused surface by phase-transition of paraffin. ${ }^{29-31}$ ). This indicates that surface chemical properties of paraffin remain unchanged upon heating. If the surface chemical properties are changed by heating, an obvious change of wettability should be observed on a flat surface. For instance, the CAs on a flat surface coated with PNIPAAm change from $\sim 63^{\circ}$ to $\sim 94^{\circ}$ at higher temperature than LCST (around $32{ }^{\circ} \mathrm{C}$ ). ${ }^{32}$ The OTMS-modified TNR brush, that is uncoated with paraffin, also exhibited the same CA irrespective of substrate temperature. Consequently, the drastic change of wettability for the paraffin/TNR brush is attributed to surface roughness change accompanied by phasetransition of paraffin by heating/cooling, while the surface chemical properties remain unchanged. The thermal expansion of paraffin on the TNR brush resulted in a decrease of surface roughness of the paraffin/TNR brush, which makes the surface less hydrophobic above $50{ }^{\circ} \mathrm{C}$. Indeed, a decrease of surface roughness at higher temperature was observed by atomic force microscope (AFM) investigations (see Fig. S5 $\dagger$ ). The transition temperature of $50{ }^{\circ} \mathrm{C}$ was slightly higher than the melting point of paraffin $\left(42-44^{\circ} \mathrm{C}\right)$, that is presumably because the accurate temperature of the paraffin coating is lower than the temperature of the substrate due to a large paraffin/air interfacial area. Most of stimuli-responsive wettability reported so far rely on changes of surface chemical properties of materials induced by external stimuli. ${ }^{\mathbf{1 1 8}}$ The results shown here clearly demonstrate the fabrication of thermo-responsive wettability by surface roughness change accompanied by phase-transition of paraffin.

One of potential applications of the surfaces with reversibly responsive wettability is a droplet transport. ${ }^{18}$ Precise control over the motion of tiny amount of droplets on a solid surface is of importance in a variety of applications including biochemical analysis, ${ }^{33}$ droplet microfluidics, ${ }^{34}$ self-cleaning, ${ }^{35}$ and drug delivery. ${ }^{36}$ To realize the droplet transport, a spatial gradient of wettability on a surface is required to drive the droplet in which the droplet can move toward the more wettable parts of the surface. The wettability gradient force is expressed as

$$
F=\pi R^{2} \gamma \frac{\mathrm{d} \cos \theta}{\mathrm{d} x}
$$

where $R$ and $\gamma$ represent the base radius and surface tension of the droplet, respectively. $\mathrm{d} \cos \theta / \mathrm{d} x$ represents the spatial gradient of wettability as $\theta$ and $x$ represent contact angle and the length of the substrate..$^{17,29}$ The paraffin/TNR brush exhibits higher hydrophobicity with a CA of $\sim 157^{\circ}$ below $50{ }^{\circ} \mathrm{C}$ (at room temperature), and at temperature above $50{ }^{\circ} \mathrm{C}$ the surface drastically changes to a less hydrophobic state with a CA of $\sim 118^{\circ}$. In this system, a droplet transport can be achieved by a spatial heating of the paraffin/TNR brush at room temperature as the temperature gradient applied on the substrate yields a drastic wettability gradient at the point with around $50{ }^{\circ} \mathrm{C}$; the droplet moves toward spatially heating parts. Although unidirectional droplet transport has been demonstrated on a static wettability gradient surface induced by systematic variation of microscale structures (a surface roughness gradient), ${ }^{37}$ reversible change of wettability of the paraffin/TNR brush allows for a multi-directional droplet transport using the same sample repeatedly. To demonstrate a multi-directional droplet transport, water droplets were dropped onto a position where the substrate was spatially heated at temperature higher than $50{ }^{\circ} \mathrm{C}$ (Fig. 3). Fig. 3a shows an experimental setup. The back side of the paraffin/TNR brush was heated along the red line. A temperature profile on the paraffin/TNR brush was measured by an infrared thermography camera (Fig. 3b). As observed in the temperature profile, temperature at the portion in contact with the heater was about $52{ }^{\circ} \mathrm{C}$ and the temperature gradient to surrounding was $1.5{ }^{\circ} \mathrm{C} \mathrm{mm}^{-1}$. A water droplet (15 $\mu \mathrm{L}$ ) was dripped $3 \mathrm{~mm}$ away from the heated part (a point indicated by a blue pentagon) of which temperature was around $47^{\circ} \mathrm{C}$. Fig. 3c shows the snapshots of a droplet transport on the paraffin/TNR brush. The water droplet moved rapidly to the heated part at a velocity of $\sim 18 \mathrm{~mm} \mathrm{~s}^{-1}$. Although the transport velocity was lower than that reported on the PNIPAAm-grafted structured PDMS,,$^{32}$ the velocity of $\sim 18 \mathrm{~mm} \mathrm{~s}^{-1}$ observed on the paraffin/TNR brush is still higher than those reported so far, such as spindle knot structure coated with PNIPAAm (about 0.1 $\mu \mathrm{m} \mathrm{s}{ }^{-1}$ ), polymer-coated $\mathrm{TiO}_{2}$ nanorods (a few $\mu \mathrm{m} \mathrm{s} \mathrm{s}^{-1}$ ) and photoisomerizable monolayer-coated $\mathrm{SiO}_{2}$ (about $1 \mathrm{~mm}$ $\left.\mathrm{s}^{-1}\right) \cdot{ }^{38-40}$ The fast droplet transport is probably due to the fact that the large contact angle changes with a temperature difference of a few degrees at around $50{ }^{\circ} \mathrm{C}$; a large wettability gradient force was generated by the large $\mathrm{d} \cos \theta / \mathrm{d} x$ on a substrate with a temperature gradient. On the same substrate, it is possible to transport the droplets in different directions with almost same velocity by changing the location of heating (Fig. 3d). As demonstrated here, water droplets can move to the heated parts on the paraffin/TNR brush at room temperature. By using same experimental set up, transport and interfusion of

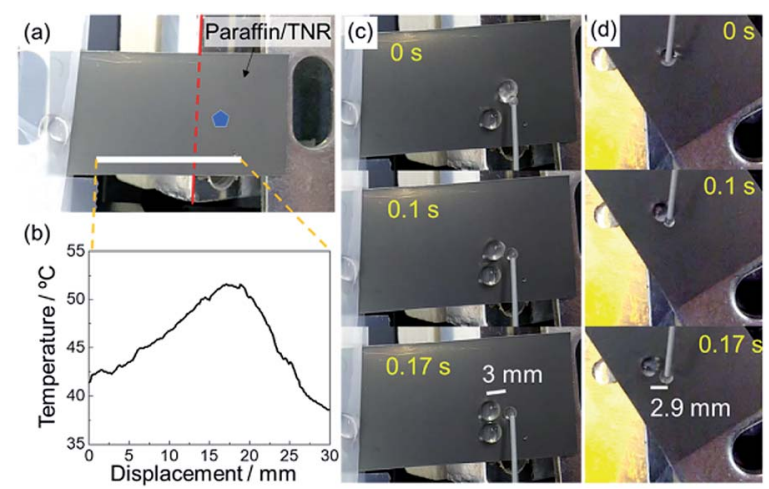

Fig. 3 Droplet transport on the paraffin/TNR brush. (a) Experimental setup where the back side of the paraffin/TNR brush was heated along the red line. The blue pentagon indicates the point where water droplet $(15 \mu \mathrm{L})$ falls. (b) A temperature profile on the paraffin/TNR brush that was measured on the white bar of the image (a). The snapshots of droplet transport on the paraffin/TNR brush in (c) the experimental setup of (a) and (d) the different configuration where the heater was $45^{\circ}$ rotated in a counter clockwise fashion. 
tiny amount of two droplets are also demonstrated (see Fig. S6†). Two different droplets moved to the heated part and then merged together. On the tilted substrate, a collection of the mixed droplet was also possible (see Fig. S7†). Although extended transport distances are required for practical applications, this fast and multi-directional droplet transport system based on surface roughness change is expected for the transport of droplets containing rare molecules that are sensitive to functional groups at the interface (e.g., biomolecules). ${ }^{18,37,41}$

\section{Conclusions}

We demonstrated thermo-responsive wettability induced by surface roughness change on titanate nanorod brushes that were coated with paraffin (paraffin/TNR brush). The paraffin/ TNR brush exhibited thermo-responsive and reversible wettability change in a hydrophobic regime: superhydrophobicity with a CA of $\sim 157^{\circ}$ below $50{ }^{\circ} \mathrm{C}$, a less hydrophobic state with a CA of $\sim 118^{\circ}$ above $50{ }^{\circ} \mathrm{C}$. The reversible and large wettability change was attributed to surface roughness change induced by thermal expansion of paraffin accompanied by phasetransition, while the surface chemical properties remained unchanged. A spatial heating of the paraffin/TNR brush at room temperature allowed for a fast and multi-directional droplet transport owing to the reversible and large wettability change. The present approach for the fabrication of a surface with stimuli-responsive wettability based on the expansion of paraffin will be applied to other substances that can change the volume by external stimuli, which will promote scientific advancement and technology innovation in the field of smart surface.

\section{Conflicts of interest}

There are no conflicts to declare.

\section{Acknowledgements}

The present work is partially supported by Grand-in-Aids from the Ministry of Education, Culture, Sports, Science and Technology (MEXT), administrated by Japan Society for the Promotion of Science (JSPS), and also by JST, PRESTO grant number JPMJPR19I3, Japan.

\section{Notes and references}

1 Y. Liu, L. Mu, B. Liu and J. Kong, Chem.-Eur. J., 2005, 11, 2622-2631.

2 F. Xia, Y. Zhu, L. Feng and L. Jiang, Soft Matter, 2009, 5, 275281.

3 X. Yao, Y. Hu, A. Grinthal, T.-S. Wong, L. Mahadevan and J. Aizenberg, Nat. Mater., 2013, 12, 529-534.

4 D. P. Regan and C. Howell, Curr. Opin. Colloid Interface Sci., 2019, 39, 137-147.

5 K. Liu, M. Cao, A. Fujishima and L. Jiang, Chem. Rev., 2014, 114, 10044-10094.
6 M. A. C. Stuart, W. T. S. Huck, J. Genzer, M. Müller, C. Ober, M. Stamm, G. B. Sukhorukov, I. Szleifer, V. V. Tsukruk, M. Urban, F. Winnik, S. Zauscher, I. Luzinov and S. Minko, Nat. Mater., 2010, 9, 101-113.

7 W. Jiang, G. Wang, Y. He, X. Wang, Y. An, Y. Song and L. Jiang, Chem. Commun., 2005, 3550-3552.

8 Z. Cheng, H. Lai, Y. Du, K. Fu, R. Hou, C. Li, N. Zhang and K. Sun, ACS Appl. Mater. Interfaces, 2014, 6, 636-641.

9 X. Yu, Z. Wang, Y. Jiang, F. Shi and X. Zhang, Adv. Mater., 2005, 17, 1289-1293.

10 J. Lahann, S. Mitragotri, T. N. Tran, H. Kaido, J. Sundaram, I. S. Choi, S. Hoffer, G. A. Somorjai and R. Langer, Science, 2003, 299, 371-374.

11 G. Hou, M. Cao, C. Yu, S. Zheng, D. Wang, Z. Zhu, W. Miao, Y. Tian and L. Jiang, ACS Appl. Mater. Interfaces, 2017, 9, 23238-23245.

12 Z. Lei, G. Zhang, Y. Deng and C. Wang, ACS Appl. Mater. Interfaces, 2017, 9, 8967-8974.

13 B. Xin and J. Hao, Chem. Soc. Rev., 2010, 39, 769-782.

14 L. Chen, T. Yang, Y. Niu, X. Mu, Y. Gong, Y. Feng, N. F. De Rooij, Y. Wang, H. Li and G. Zhou, Chem. Commun., 2020, 56, 2837-2840.

15 W. Zhang, N. Liu, Q. Zhang, R. Qu, Y. Liu, X. Li, Y. Wei, L. Feng and L. Jiang, Angew. Chem., Int. Ed., 2018, 57, 5740-5745.

16 T. Sun, G. Wang, L. Feng, B. Liu, Y. Ma, L. Jiang and D. Zhu, Angew. Chem., Int. Ed., 2004, 43, 357-360.

17 Y. Hou, B. Xue, S. Guan, S. Feng, Z. Geng, X. Sui, J. Lu, L. Gao and L. Jiang, NPG Asia Mater., 2013, 5, e77.

18 R. Malinowski, I. P. Parkin and G. Volpe, Chem. Soc. Rev., DOI: $10.1039 /$ d0cs00268b.

19 A. Marmur, Langmuir, 2003, 19, 8343-8348.

20 G. Whyman, E. Bormashenko and T. Stein, Chem. Phys. Lett., 2008, 450, 355-359.

21 A. B. D. Cassie and S. Baxter, Trans. Faraday Soc., 1944, 40, 546-551.

22 D. Bonn, J. Eggers, J. Indekeu and J. Meunier, Rev. Mod. Phys., 2009, 81, 739-805.

23 A. Mann, T. Germann, M. Ruiter and P. Groche, Mater. Des., 2020, 190, 108580.

24 L. Klintberg, M. Svedberg, F. Nikolajeff and G. Thornell, Sens. Actuators, A, 2003, 103, 307-316.

25 N. Kabei, M. Kosuda, H. Kagamibuchi, R. Tashiro, H. Mizuno, Y. Ueda and K. Tsuchiya, JSME Int. J., Ser. C, 1997, 40, 736-742.

26 K. Okada, Y. Tokudome and M. Takahashi, J. Sol-Gel Sci. Technol., 2016, 79, 389-394.

27 K. Okada, Y. Tokudome, P. Falcaro, Y. Takamatsu, A. Nakahira and M. Takahashi, Chem. Commun., 2012, 48, 6130-6132.

28 Y. Tokudome, K. Okada, A. Nakahira and M. Takahashi, J. Mater. Chem. A, 2014, 2, 58-61.

29 M. Villegas, Y. Zhang, N. A. Soleymani and T. F. Didar, ACS Nano, 2019, 13, 8517-8536.

30 C. Howell, A. Grinthal, S. Sunny, M. Aizenberg and J. Aizenberg, Adv. Mater., 2018, 30, 1802724. 
31 X. Lou, Y. Huang, X. Yang, H. Zhu, L. Heng and F. Xia, Adv. Funct. Mater., 2020, 30, 1901130.

32 T. N. Banuprasad, T. V. Vinay, C. K. Subash, S. Varghese, S. D. George and S. N. Varanakkottu, ACS Appl. Mater. Interfaces, 2017, 9, 28046-28054.

33 M. J. Jebrail, M. S. Bartsch and K. D. Patel, Lab Chip, 2012, 12, 2452-2463.

34 Y. Cui, D. Li and H. Bai, Ind. Eng. Chem. Res., 2017, 56, 48874897.

35 R. Blossey, Nat. Mater., 2003, 2, 301-306.

36 R. Riahi, A. Tamayol, S. A. M. Shaegh, A. M. Ghaemmaghami, M. R. Dokmeci and A. Khademshosseini, Curr. Opin. Chem. Eng., 2015, 7, 101-112.
37 A. Shastry, M. J. Case and K. F. Böhringer, Langmuir, 2006, 22, 6161-6167.

38 Y. Hou, L. Gao, S. Feng, Y. Chen, Y. Xue, L. Jiang and Y. Zheng, Chem. Commun., 2013, 49, 5253-5255.

39 F. V. Monteleone, G. Caputo, C. Canale, P. D. Cozzoli, R. Cingolani, D. Fragouli and A. Athanassiou, Langmuir, 2010, 26, 18557-18563.

40 K. Ichimura, S. K. Oh and M. Nakagawa, Science, 2000, 288, 1624-1626.

41 B. L. Ekerdt, C. M. Fuentes, Y. Lei, M. M. Adil, A. Ramasubramanian, R. A. Segalman and D. V. Schaffer, Adv. Healthcare Mater., 2018, 7, 1800225. 